\title{
Cosmological Evolution of X-ray Selected AGNs and Synthesis of the X-ray Background
}

\author{
Yoshihiro Ueda ${ }^{1}$, Masayuki Akiyama ${ }^{2}$, Günther Hasinger ${ }^{3}$, \\ Takamitsu Miyaji ${ }^{4}$ and Michael G. Watson ${ }^{5}$ \\ ${ }^{1}$ Department of Astronomy, Kyoto University, Japan \\ email: ueda@kusastro.kyoto-u.ac.jp \\ ${ }^{2}$ Astronomical Institute, Tohoku University, Japan \\ ${ }^{3}$ Institute for Astronomy, University of Hawaii, USA \\ ${ }^{4}$ Instituto de Astronomía, Universidad Nacional Autónoma de México, Mexico \\ ${ }^{5}$ Department of Physics and Astronomy, University of Leicester, UK
}

\begin{abstract}
X-ray surveys provide us with one of the least biased samples of Active Galactic Nuclei (AGNs) against obscuration. Here we present the most up-to-date AGN X-ray luminosity function (XLF) and absorption function over the redshift range from 0 to 5 , using the largest, highly complete sample ever available obtained from surveys of various depth, depth, and energy bands. We utilize a maximum likelihood method to reproduce the count-rate versus redshift distribution for each survey, by taking into account the evolution of the absorbed fraction, contribution from Compton-thick AGNs, and AGN broad band X-ray spectra including reflection components from tori based on the luminosity and redshift dependent unified scheme. We find that the shape of the XLF at $z \sim 1-3$ is significantly different from that in the local universe, for which the luminosity dependent density evolution (LDDE) model gives the best description. These results establish the standard population synthesis model of the X-Ray Background (XRB), which well reproduces the source counts in both soft and hard bands, the observed fractions of Compton-thick AGNs, and the spectrum of the XRB.
\end{abstract}

Keywords. galaxies: active, X-rays: diffuse background

\section{Introduction}

The majority of AGNs are obscured by gas and dust surrounding the supermassive black holes (SMBHs), being classified as "type-2" AGNs. To elucidate the growth history of SMBHs, a complete survey of AGNs including heavily obscured populations throughout the history of the universe is necessary. X-ray observations, in particular those at high energies above a few $\mathrm{keV}$, provide one of the most powerful approach for AGN detection thanks to the strong penetrating power against absorption and little contamination from star lights in the host galaxies. Furthermore, the deepest X-ray surveys currently available achieve the highest sensitivity even for unobscured ("type-1") AGNs among those at any wavelengths (e.g., Brandt \& Hasinger 2005).

The integration of emission from all accreting SMBHs in the universe is observed as the X-ray background (XRB). To quantitatively solve the XRB origin is equivalent to revealing the cosmological evolution of AGNs that constitute the XRB. It is very important to construct a "population synthesis model" of the XRB, where evolutions of all X-ray emitting AGNs with various types are formulated (for previous works, see e.g. Comastri et al. 1995; Ueda et al. 2003; Ballantyne, Everett, \& Murray 2006; Gilli, Comastri, \& Hasinger 2007; Treister, Urry, \& Virani 2009). Two major elements in the 
population synthesis models are the X-ray luminosity function (XLF) and absorption function (or $N_{\mathrm{H}}$ function) of AGNs.

This paper gives a digest of our recent work on the AGN XLF and population synthesis model of the XRB (Ueda et al. 2014). We determine an AGN XLF over the redshift range from 0 to 5 by utilizing one of the largest combined samples ever available, obtained from surveys performed with Swift/BAT, the Monitor of All-sky X-ray Image (MAXI), ASCA, XMM-Newton, Chandra, and ROSAT. The sample consists of 4039 detections in the soft (0.5-2 keV) and/or hard $(>2 \mathrm{keV})$ bands. We utilize a maximum likelihood method to reproduce the count-rate versus redshift distribution for each survey, by taking into account the selection biases. In the analysis, the contribution of Compton-thick AGNs is considered, which is found to be more important in harder bands and at fainter fluxes. This enables us to determine the intrinsic XLF and the absorption function of type-1 plus type-2 AGNs with an unprecedented accuracy, thus establishing a population synthesis model of the XRB that is consistent with most of observational constraints currently available.

\section{Sample}

In order to investigate the XLF and absorption function of AGNs covering a wide range of luminosity and redshift, it is vital to construct a sample combined from various surveys with different flux limits and area. Also, high degrees of identification completeness in terms of spectroscopic and/or photometric redshift determination are required to minimize systematic uncertainties caused by sample incompleteness. X-ray surveys at higher energies are more suitable to detect obscured AGNs with less biases. Nevertheless, those in the soft band $(\approx 0.5-2 \mathrm{keV})$ are also quite useful as long as such biases are properly corrected.

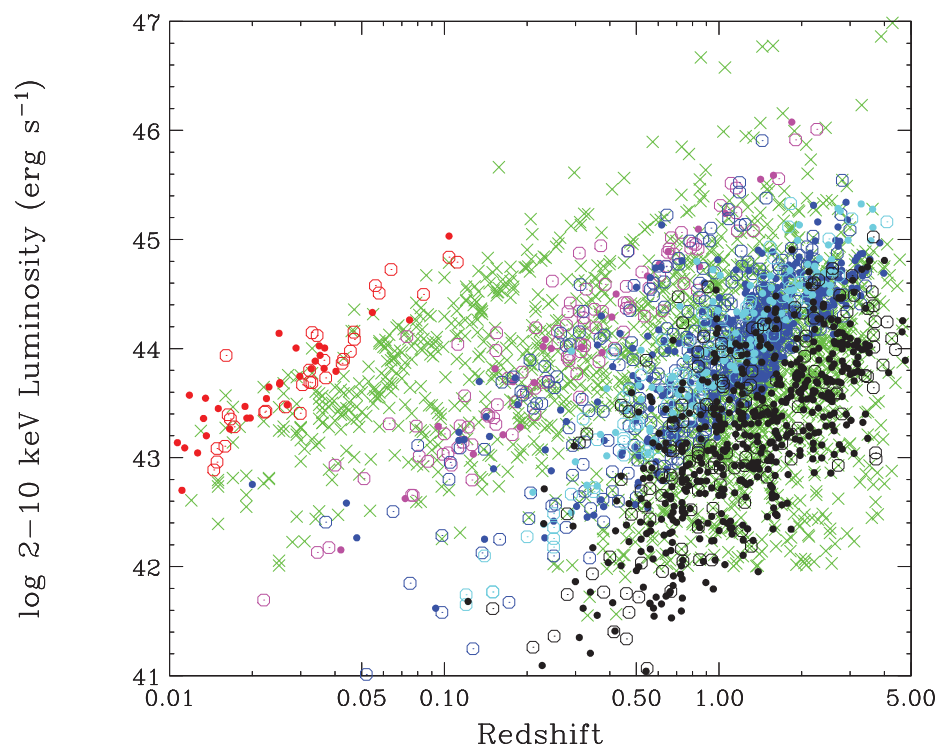

Figure 1. Redshift-luminosity plot of our sample. The open and filled circles represent X-ray type-1 AGNs (with $\log N_{\mathrm{H}}<22$ ) and X-ray type-2 AGNs (with $\log N_{\mathrm{H}} \geqslant 22$ ), respectively, detected in the hard $(>2 \mathrm{keV})$ band (red: Swift/BAT sample, magenta: $A S C A$ sample, blue: XMM-Newton sample, cyan: CLASXS and CLANS sample, black: CDFN and CDFS sample). Those detected in the soft $(0.5-2 \mathrm{keV})$ band are marked by crosses (green). 
Our sample is composed of those from the Swift/BAT 9-month survey, MAXI 7month survey, ASCA Medium Sensitivity Survey (AMSS) and Large Sky Survey (ALSS), Subaru/XMM-Newton Deep Survey (SXDS), XMM-Newton survey of the Lockman Hole, HELLAS2XMM survey, Hard Bright Serendipitous Sample in the XMM-Newton Bright Survey, Chandra Large Area Synoptic X-ray Survey (CLASXS), Chandra Lockman Area North Survey (CLANS), Chandra Deep Survey North (CDFN) and South (CDFS), and various ROSAT surveys. For details, see Ueda et al. (2014) and references therein.

For convenience, we calculate an intrinsic (de-absorbed) luminosity in the rest-frame 2-10 keV band, $L_{\mathrm{X}}$, for each object. To convert the count rate into $L_{\mathrm{X}}$, we consider the absorption column density $\left(N_{\mathrm{H}}\right)$ of each source from the observed spectrum or hardness ratio, by taking into account with the energy response of the instrument used in the survey. Figure 1 displays the $L_{\mathrm{X}}$ versus redshift distribution for the hard band and soft band samples.

\section{Evolution of Absorbed AGN Fraction}

Utilizing the samples of Swift/BAT, AMSS, and SXDS, we calculate the fraction of absorbed Compton-thin AGNs (i.e., $\log N_{\mathrm{H}}=22-24$ ) in total Compton-thin AGNs (i.e., $\left.\log N_{\mathrm{H}}=20-24\right)$ in different redshift and luminosity ranges. The results plotted in figure 2 . As noticed, we confirm the strong anti-correlation between the absorption fraction and luminosity. In addition, a redshift dependence is clearly seen that the absorption fraction becomes larger at higher redshift by keeping the similar anti-correlation with luminosity. This is fully consistent with Hasinger (2008). From our analysis of the full sample, we find that the absorption fraction among Compton-thin AGNs at $\log L_{\mathrm{X}}=43.75$ is proportional to $(1+z)^{0.48 \pm 0.03}$ up to $z=2$.

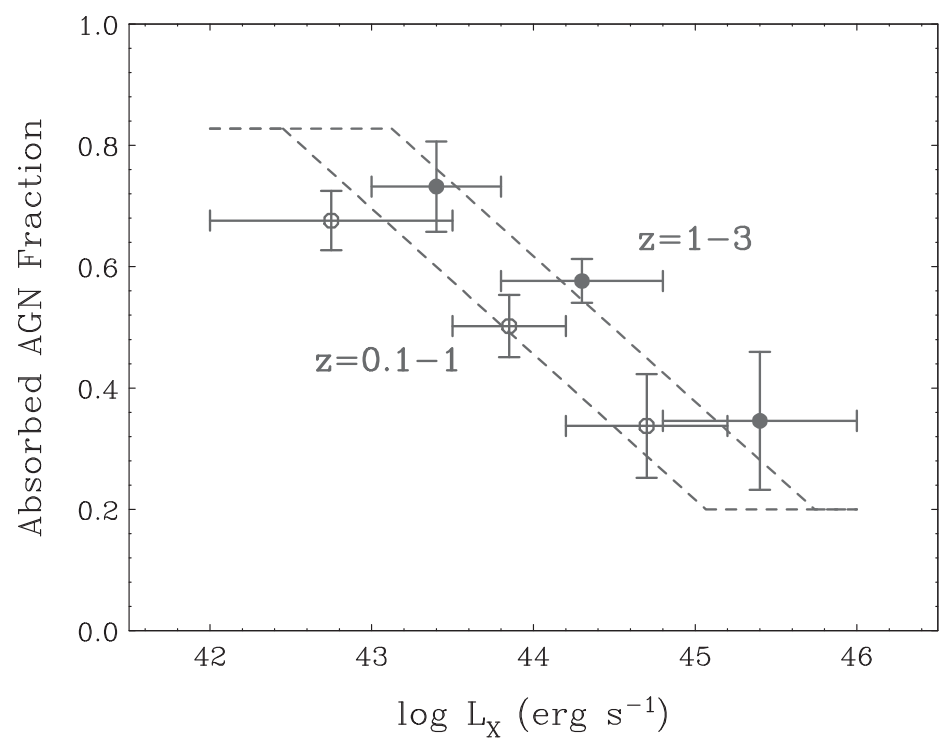

Figure 2. Absorbed fraction among Compton-thin AGNs plotted against luminosity at $z=0.1-1$ (blue) and $z=1-3$ (red) as determined from the Swift/BAT, AMSS, and SXDS (hard band) samples. The dashed lines represent the best-fit models calculated at the mean redshifts for the $z=0.1-1$ and $z=1-3$ samples. 


\section{X-ray Luminosity Function}

The AGN XLF, $d \Phi_{\mathrm{X}}\left(L_{\mathrm{X}}, z\right) / d \log L_{\mathrm{X}}$, represents the number density per unit co-moving volume per $\log L_{\mathrm{X}}$ as a function of $L_{\mathrm{X}}$ and $z$ in units of $\mathrm{Mpc}^{-3} \mathrm{dex}^{-1}$. We develop a new analysis method to utilize the list of the count rate and redshift obtained in each survey, the most basic observational quantities without any corrections. This approach is based on a "forward method", where comparison between the prediction and observation is made at the final stage. Namely, with a maximum likelihood algorithm we search for a set of parameters of the XLF and absorption function that best reproduce the count-rate versus $z$ distributions of all surveys used in the analysis. A merit is that we can properly take into account any complex X-ray spectra of AGNs including Compton-thick ones.

We find that the shape of the XLF at $z=1-3$ is quite different from that in the local universe in the sense that the slope at the low luminosity range is significantly flatter than those observed at lower redshifts. This trend can be much better reproduced by the luminosity dependent density evolution (LDDE) model than the luminosity and density evolution (LADE) model (Aird et al. 2010), where a constant relative shape of the XLF in the logarithmic scales is assumed over the full redshift range.

Figure 3 plot the co-moving space number density of Compton-thin AGNs as a function of redshift integrated in different luminosity bins, $\log L_{\mathrm{X}}=42-43,43-44,44-45$, and 4547. One can clearly confirm the global "down-sizing" evolution, where more luminous AGNs have their number density peak at higher redshifts compared with less luminous ones (e.g., Ueda et al. 2003; Hasinger, Miyaji, \& Schmidt 2005). We note that when we only focus attention on the high redshift range of $z \gtrsim 3$, our LDDE model indicates an "up-sizing" evolution instead (i.e., the number ratio of less luminous AGNs to more luminous ones is larger at earlier epochs).

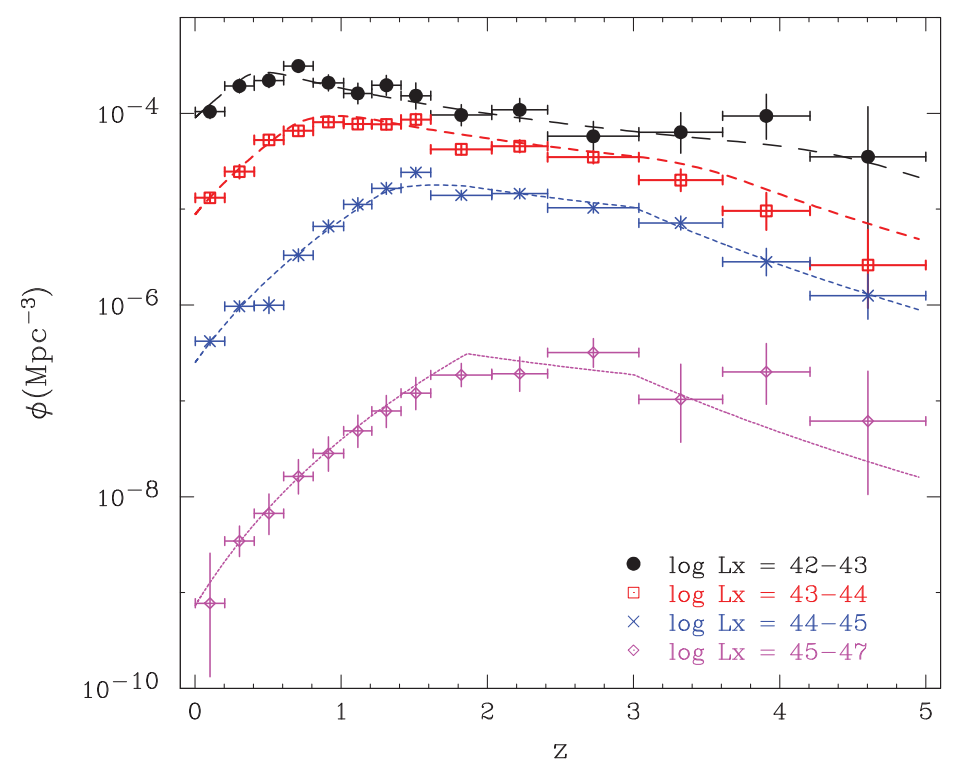

Figure 3. Comoving number density of AGNs plotted against redshift in different luminosity bins (Compton-thin AGNs only). The curves are our best-fit model, and the data points are based on the " $N^{\text {obs }} / N^{\text {mdl }}$ method" with $1 \sigma$ errors. To ensure independence of the plotted data, we only plot the hard-band samples in the region of $z<2$ and $\log L_{\mathrm{X}}<44$, and the soft-band samples for the rest. 


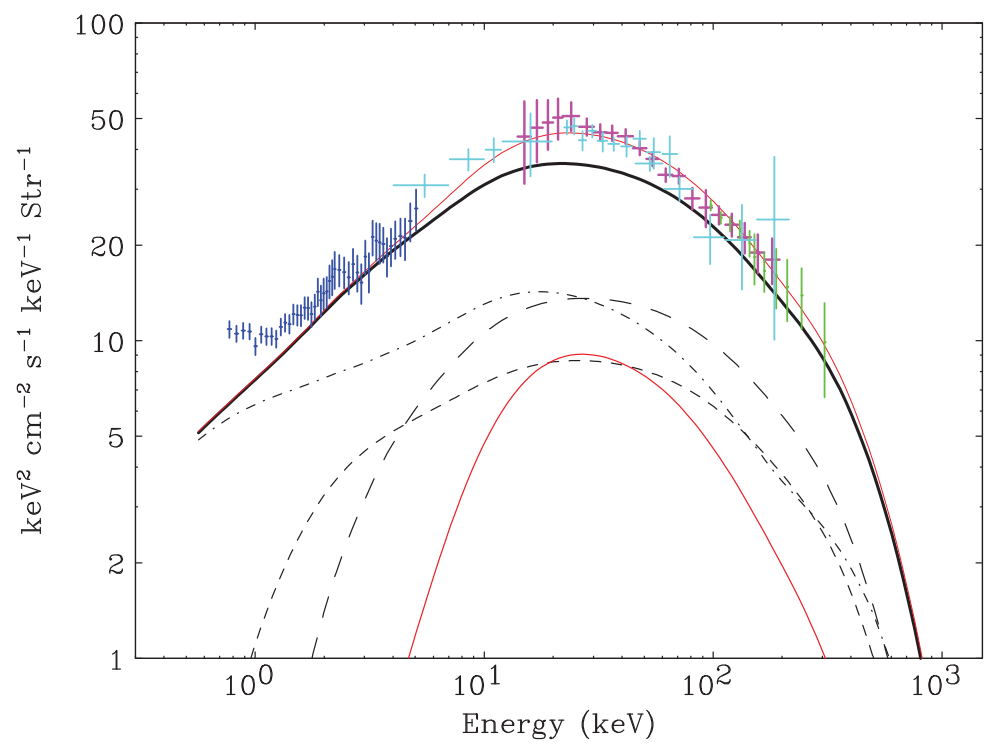

Figure 4. XRB spectrum calculated from our AGN population synthesis model (upper solid curve, red) compared with the observed data by various missions (Ajello et al. 2008). Middle solid curve (black): the integrated spectrum of Compton-thin AGNs (log $\left.N_{\mathrm{H}}<24\right)$. Lower solid curve (red): that of Compton-thick AGNs ( $\left.\log N_{\mathrm{H}}=24-26\right)$. Long-dashed curve (black): that of AGNs with $\log N_{\mathrm{H}}=23-24$. Short-dashed curve (black): that of AGNs with $\log N_{\mathrm{H}}=22-23$. Dot-dashed curve (black): that of AGNs with $\log N_{\mathrm{H}}<22$.

\section{Standard Population Synthesis Model of the XRB}

The combination of our best-fit XLF, absorption function, and photon index function with the template broad-band spectra of AGNs enables us to establish a new population synthesis model of the XRB. Figure 4 shows the integrated broad band spectrum of the whole AGNs at $z=0.002-5.0$ with $\log L_{\mathrm{X}}=41-47$ predicted from our model. The data points represent the measurements of the XRB observed with various missions $\mathrm{A}$ good agreement is confirmed between the model prediction and the hard XRB, supporting the overall validity of our model. In our baseline model, we assume that the number ratio of Compton-thick AGNs $\left(\log N_{\mathrm{H}}=24-26\right)$ to the absorbed Compton-thin AGNs $\left(\log N_{\mathrm{H}}=\right.$ 22-24) is unity, by assuming that they follow the same evolution as Compton-thin AGNs. This ratio can be constrained to be $\approx 0.5-1.6$ to produce the $20-50 \mathrm{keV}$ XRB intensity within present uncertainties.

Figure 5 shows the fractions of Compton-thick AGNs $\left(\log N_{\mathrm{H}}=24-26\right)$ in the total AGNs $\left(\log N_{\mathrm{H}} \leqslant 26\right)$ as a function of flux predicted from surveys in the $2-10 \mathrm{keV}$ and $10-40 \mathrm{keV}$ bands. The Compton-thick AGN fraction reaches $\approx 20 \%$ at $S \sim 10^{-16} \mathrm{erg}$ $\mathrm{cm}^{-2} \mathrm{~s}^{-1}$ in the $2-10 \mathrm{keV}$ band, the flux limit of Chandra deep surveys. We find that the observed Compton-thick AGN fractions at various flux limits in the $2-10 \mathrm{keV}$ (or 0.5-8 keV) band are in good agreements with the model prediction. In the $10-40 \mathrm{keV}$ band, our model is consistent with the observed Compton-thick fraction at $S \sim 10^{-11}$ erg $\mathrm{cm}^{-2} \mathrm{~s}^{-1}$ observed by the Swift/BAT 9-month survey and with the upper limit $(<0.23$ at a $90 \%$ confidence level) obtained from the first $N u S T A R$ extragalactic survey. In our baseline model, the intrincic fraction of Compton-thick AGNs among the whole AGNs at $\log L_{\mathrm{X}}=43.75$ is $30 \pm 2 \%$ at $z=0,37 \pm 2 \%$ at $z=1$, and $42 \pm 2 \%$ at $z \geqslant 2$. They are fully consistent with the results obtained by Brightman \& Ueda (2012) from the CDFS data at $z>0.1$. 


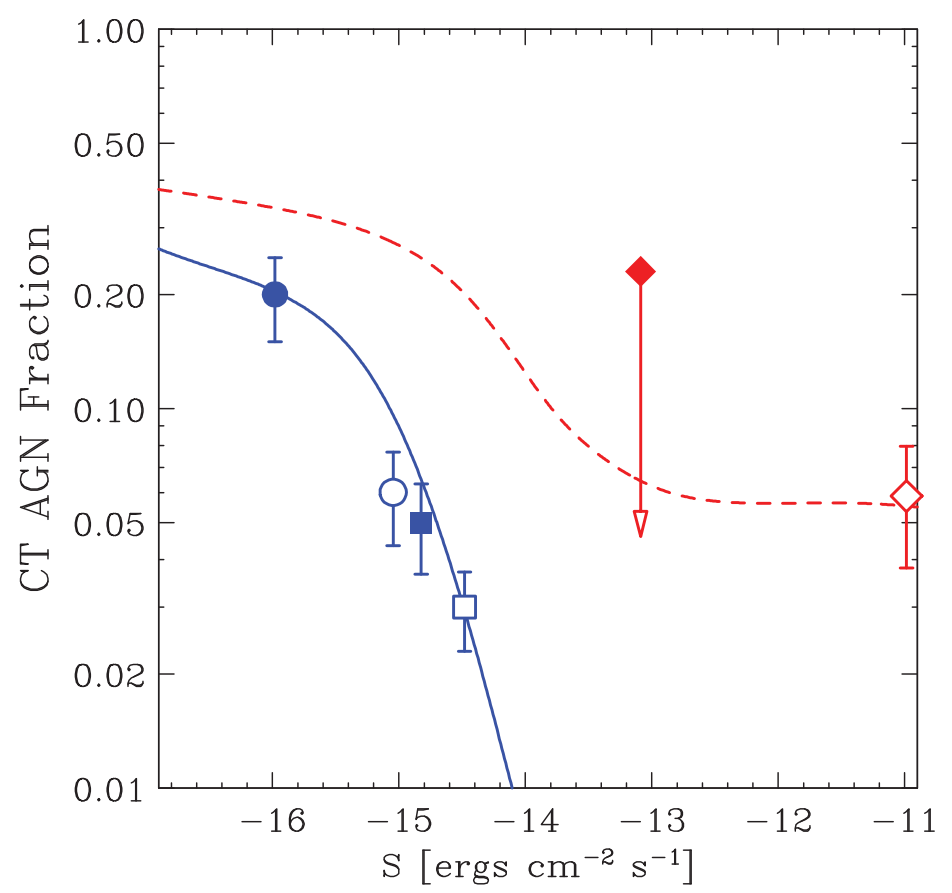

Figure 5. Fractions of Compton-thick AGNs $\left(\log N_{\mathrm{H}}=24-26\right)$ in the total AGNs given as a function of flux in the 2-10 keV band (solid blue) and 10-40 keV band (dashed, red) predicted from our baseline model. The data points correspond to the observed Compton-thick AGN fractions by Brightman \& Ueda (2012) (filled circle), Brunner et al. (2008) (open circle), Tozzi et al. (2006) (filled square), and Hasinger et al. (2007) (open square). The arrow (filled diamond) denotes the $90 \%$ confidence upper limit of the Compton-thick fraction obtained by NuSTAR in the 8-24 keV band (Alexander et al. 2013), and the open diamond is that from the Swift/BAT 9-month survey in the 14-195 keV band (Tueller et al. 2008; Ichikawa et al. 2012). The fluxes are converted into the $10-40 \mathrm{keV}$ band by assuming a photon index of 1.8 .

Thus, our baseline model may be regarded as the standard population synthesis model of the XRB, which well reproduces the source counts in both soft and hard bands, the observed fractions of Compton-thick AGNs, and the spectrum of the XRB. The biggest advantage is that it utilizes the most precise XLF and absorption function that depends both on luminosity and redshift. Our model also takes into account the broad band spectra including the reflection components from the tori based on the "luminosity and redshift dependent unified scheme" as well as the photon index distributions that are different between type-1 and type-2 AGNs. The entire analysis has been performed selfconsistently on the basis of these assumptions.

These results lead us to derive a bolometric luminosity function of AGNs by considering the luminosity-dependent bolometric correction factor. We find that the luminosity density of the whole AGNs has a peak around $z \sim 2$, where AGNs with bolometric luminosities of $\log L=46-47$ make the largest contribution. On the basis of Soltan's argument, the local mass density of supermassive black holes (SMBHs) is reproduced by adopting an averaged AGN radiation efficiency of $\simeq 0.08$. More discussions on the evolution of SMBH mass function can be found in Ueda et al. (2014).

\section{References}

Aird, J. et al. 2010, MNRAS, 401, 2531 
Ajello, M. et al. 2008, ApJ, 689, 666

Alexander, D. M. et al. 2013, ApJ, 773, 125

Ballantyne, D. R., Everett, J. E., \& Murray, N. 2006, ApJ, 639, 740

Brandt, W. N. \& Hasinger, G. 2005, ARAA, 43, 827

Brightman, M. \& Ueda, Y. 2012, MNRAS, 423, 702

Brunner, H. et al. 2008, A\&SA, 479, 283

Comastri, A., Setti, G., Zamorani, G., \& Hasinger, G. 1995, A\&A, 296, 1

Gilli, R., Comastri, A., \& Hasinger, G. 2007, A\& $A$, 463, 79

Hasinger, G., Miyaji, T., \& Schmidt, M. 2005, A\&A, 441, 417

Hasinger, G. et al. 2007, ApJS, 172, 29

Hasinger, G. 2008, A\&̈A, 490, 905

Ichikawa, K. et al. 2012, ApJ, 754, 45

Tozzi, P. et al. 2006, A\& $A, 451,457$

Treister, E., Urry, C. M., \& Virani, S. 2009, ApJ, 696, 110

Tueller, J., et al. 2008, ApJ, 681, 113

Ueda, Y., Akiyama, M., Ohta, K., \& Miyaji, T. 2003, ApJ, 598, 886

Ueda, Y. et al. 2014, ApJ, submitted 